\title{
Ultra-Dense III-V-on-Silicon Nitride Frequency Comb Laser
}

\author{
Stijn Cuyvers ${ }^{1,2}$, Bahawal Haq ${ }^{1,2}$, Camiel Op de Beeck ${ }^{1,2}$, Stijn Poelman ${ }^{1,2}$, Artur Hermans ${ }^{1,2}$, \\ Zheng Wang ${ }^{1,2}$, Gunther Roelkens ${ }^{1,2}$, Kasper Van Gasse ${ }^{1,2}$, and Bart Kuyken ${ }^{1,2}$ \\ (1) Photonics Research Group, Ghent University - imec, Ghent, Belgium Stijn.Cuyvers@ugent.be \\ (2) Center for Nano- and Biophotonics, Ghent University, Belgium
}

\begin{abstract}
A heterogeneously integrated III-V-on-silicon nitride mode-locked laser is demonstrated. The device is fabricated by microtransfer printing an InP/InAlGaAs-based multiple-quantum-well coupon. A dense comb with a $755 \mathrm{MHz}$ repetition rate, a $1 \mathrm{~Hz}$ ASE limited RF linewidth and a $200 \mathrm{kHz}$ optical linewidth is achieved.
\end{abstract}

\section{Introduction}

Comb sources on a photonic chip have gained considerable interest in recent years for their potential in various domains, including optical ranging, microwave photonics, spectroscopy, timeand frequency metrology ${ }^{[1],[2]}$. While platforms such as quantum cascade lasers (QCLs), electrooptic and Kerr combs have shown impressive developments, their use in a number of applications remains elusive because of their large line spacing, limited number of usable comb lines and integration challenges ${ }^{[3]-[6]}$. In particular the concept of dual-comb spectroscopy in the gas-phase has proven difficult because of the need for ultradense optical frequency combs ${ }^{[3],[7]}$. As most gasses have absorption features with a linewidth on the order of $\mathrm{a} \mathrm{GHz}$, comb sources with a line spacing $<1 \mathrm{GHz}$ are in high demand to accurately sample the spectra without the need for interleaving ${ }^{[3],[8]}$. Mode-locked laser comb sources offer an attractive platform for such applications as they can provide comb spectra with ultranarrow line spacing. Moreover, these devices allow for high conversion efficiencies and can be electrically pumped, omitting the need for an external optical pump source ${ }^{[9]}$. Due to the high waveguide losses of monolithic III-V, InP, and IIIV-on-Si platforms and the difficulties with heterogeneous integration, current state-of-the-art integrated passively mode-locked lasers fail to demonstrate comb spectra with sub-GHz repetition rates ${ }^{[8],\left[{ }^{2}\right] \text {. }}$

In this work, we leverage the ultra-low losses of silicon nitride $\left(\mathrm{Si}_{3} \mathrm{~N}_{4}\right)$ to build a heterogeneously integrated III-V-on- $\mathrm{Si}_{3} \mathrm{~N}_{4}$ mode-locked laser (MLL) with a record-low repetition rate of $755 \mathrm{MHz}$. The technique of microtransfer printing is used to integrate an $\ln P / \ln A \mid G a A s$ based multiple-quantum-well (MQW) semiconductor optical amplifier (SOA) on an ultra-low-loss sili- con nitride waveguide platform, hence enabling wafer scale manufacturing. Excellent noise performance is achieved, such as an ASE limited RF linewidth of $1 \mathrm{~Hz}$ and an optical linewidth of $200 \mathrm{kHz}$.

\section{Design and fabrication}

A schematic of the MLL is depicted in Figure 1(a). An extended ring cavity geometry was employed, consisting of two $10 \mathrm{~cm} \mathrm{Si} 3 \mathrm{~N}_{4}$ spirals, deposited by means of low-pressure chemical vapor deposition on top of a silicon-on-insulator (SOI) wafer. The $\mathrm{Si}_{3} \mathrm{~N}_{4}$ waveguides were defined using deep-UV lithography and have a width of $2 \mu \mathrm{m}$ and a height of $330 \mathrm{~nm}$. To enable heterogeneous integration, a recess is locally etched in the $4.2 \mu \mathrm{m}$ silicon oxide $\left(\mathrm{SiO}_{2}\right)$ top cladding. A two-stage taper structure is employed to bridge the large index difference and ensure efficient coupling of light from the $\mathrm{Si}_{3} \mathrm{~N}_{4}$ to the III-V stack: first from the $\mathrm{Si}_{3} \mathrm{~N}_{4}$ waveguide to a silicon waveguide underneath, and subsequently to the III-V waveguide. A microscope image of the two-stage taper with transfer printed coupon is shown in Figure $1(b)$. The III-V InP/InAlGaAs layer stack is described in detail in Reference [10].

\section{Heterogeneous integration}

For the heterogeneous integration of the III-V amplifier in the recess on the passive chip, the microtransfer printing technique is used ${ }^{[10]-[12]}$. It is based on the kinetically controlled adhesion of an elastomeric stamp to pick pre-processed devices from their source substrate and print them on a target photonic integrated circuit. In contrast to bonding techniques, microtransfer printing allows to integrate a III/ $\mathrm{V}$ coupon in a recess. Moreover, this approach allows massively parallel integration, enabling wafer-scale manufacturing. After transfer printing, the coupon is post-processed to 
(a)

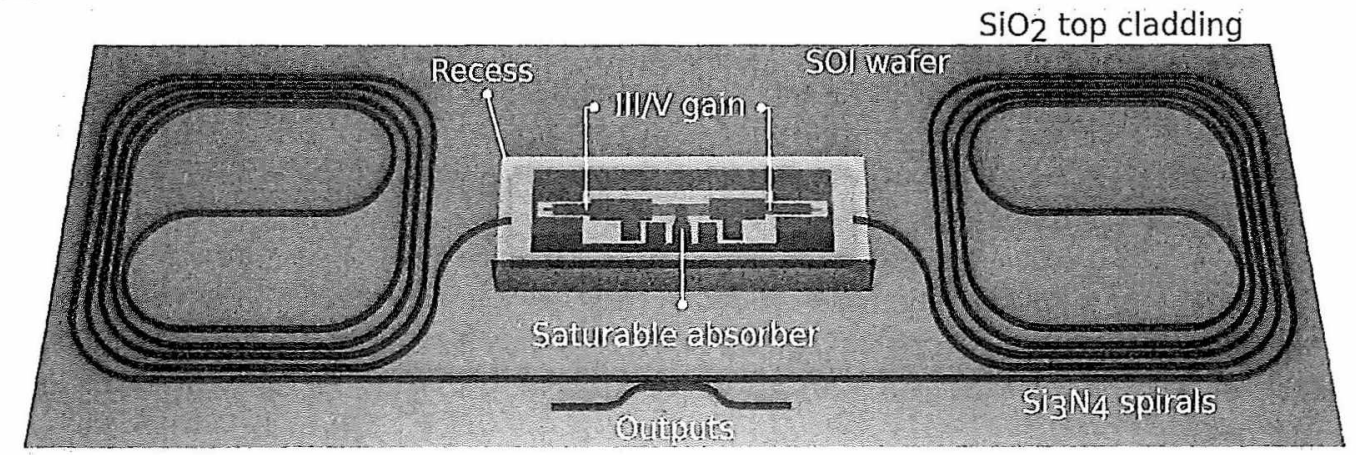

(b)

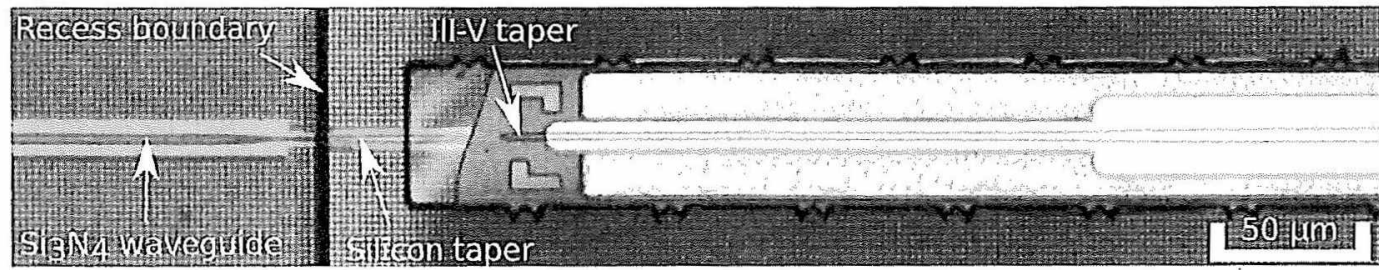

Fig. 1: (a) Schematic of the extended ring cavity mode-locked laser with $\mathrm{Si}_{3} \mathrm{~N}_{4}$ spirals and InP/InAlGaAs-based amplifiers with saturable absorber. The recess for microtransfer printing the III/N coupon is indicated. (b) Microscope image of the transfer printed coupon on top of the taper structure.

isolate a saturable absorber (SA), vias are etched to access the $\mathrm{n}$-InP layer and electrical contacts are added. The SA has a length of approximately $34 \mu \mathrm{m}$ whereas the amplifiers have a length of $600 \mu \mathrm{m}$ SOA at each side.

\section{Measurements}

The MLL was characterized on a Peltier temperature-controlled stage, which kept the device substrate at a constant temperature of $15^{\circ} \mathrm{C}$. A PGSGP probe was used for biasing, where the P-contacts were used to power the amplifiers and the S-contact was used to reverse bias the saturable absorber. Light was extracted with a single-mode fiber using a $\mathrm{Si}_{3} \mathrm{~N}_{4}$ grating coupler. Passive mode-locking at the fundamental frequency was found to occur at an SA reverse bias voltage of $-2.9 \mathrm{~V}$ with a $-0.499 \mathrm{~mA}$ SA current, and an amplifier bias of $1.88 \mathrm{~V}$ with a $75 \mathrm{~mA}$ injection current. The optical power in the fiber was measured to be around $-24 \mathrm{dBm}$, corresponding with an on-chip power of approximately $126 \mu \mathrm{W}$ when the grating coupler losses are taken into account. Although it has been shown that $10 \mu \mathrm{W}$ of optical output power suffices to perform gas-phase dual-comb spectroscopy, larger output powers could be obtained by using longer amplifiers. Figure 2(a) shows the electrical spectrum of the MLL at the aforementioned bias point, obtained with a Agilent N9010A Electrical
Spectrum Analyzer (ESA) with a $300 \mathrm{kHz}$ resolution bandwidth. A flat, densely-spaced comb spectrum is achieved with a record-low repetition rate of $755 \mathrm{MHz}$. To the best of our knowledge, this it the lowest reported repetition rate for any integrated passively mode-locked laser, enabling an unprecedented resolution for chipbased spectroscopic applications. Note that the roll-of of the RF comb at high frequencies is a consequence of the $30 \mathrm{GHz}$ bandwidth limitation of the transimpedance amplifier of the photodetector. Furthermore, the repetition frequency signal was measured with a $100 \mathrm{~Hz}$ RBW, revealing a narrow $-10 \mathrm{~dB} R F$ linewidth of $100 \mathrm{~Hz}$. The optical spectrum of the MLL was measured with an Optical Spectrum Analyzer (OSA) with a $30 \mathrm{pm}$ resolution and is depicted in Figure 2(b). A 10- $\mathrm{dB}$ optical bandwidth of $3.27 \mathrm{~nm}$ is achieved, corresponding with over 500 densely and evenly spaced comb lines. The capacity to produce such dense combs on-chip with hundreds of lines and sub-GHz linespacing is unmatched by other comb generation techniques such as QCLs, electrooptic combs and Kerr microcombs. Moreover, the spectrum of a mode-locked laser does not suffer from a strong central optical pump signal as is often the case with competing techniques. The optical linewidth was characterized by beating the MLL output with a Santec tunable laser $(60 \mathrm{kHz})$, 
(a)

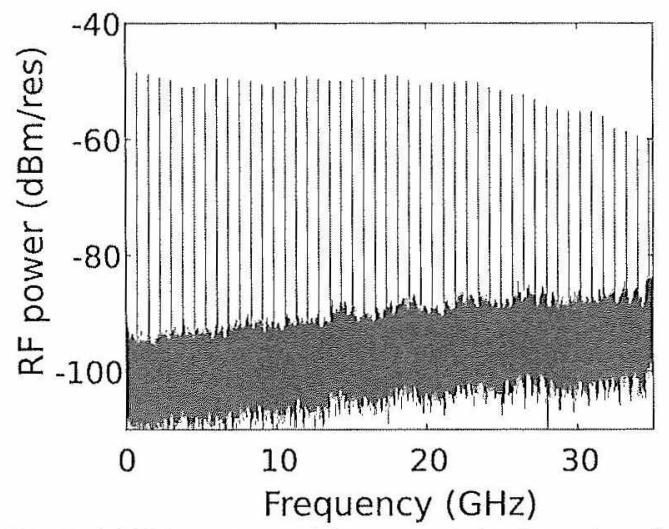

(b)

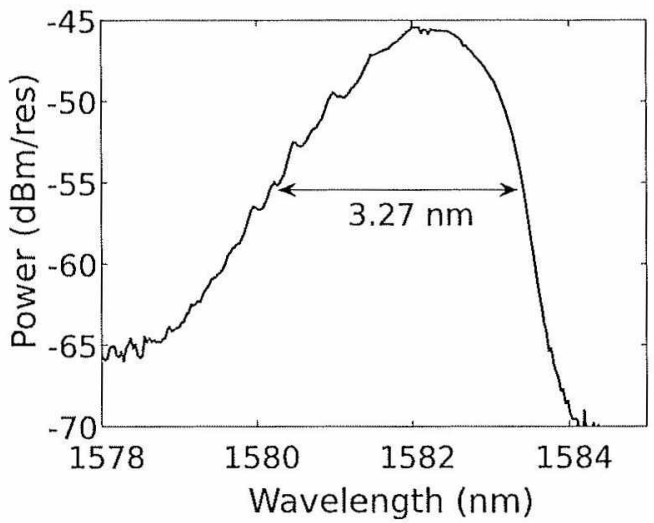

Fig. 2: (a) RF spectrum of the generated pulse train at the chosen operating point. The RBW is $300 \mathrm{kHz}$. (b) Optical spectrum measured with a $30 \mathrm{pm}$ resolution. A 10-dB optical bandwidth of $3.27 \mathrm{~nm}$ is measured.

resulting in a heterodyne beatnote with a $200 \mathrm{kHz}$ optical linewidth. Finally, single-sideband phasenoise measurements were carried out, showing a record-low $1 \mathrm{~Hz}$ ASE limited RF linewidth.

\section{Conclusions}

We have demonstrated a heterogeneously integrated III-V-on silicon nitride mode-locked laser with a record-low repetition rate of $755 \mathrm{MHz}$ and unprecedented noise performance such as a fundamental RF linewidth of $1 \mathrm{~Hz}$ and an optical linewidth of $200 \mathrm{kHz}$. The device was fabricated by microtransfer printing an InP/InAIGaAs-based multiple-quantum-well coupon on a low-loss silicon nitride platform, enabling wafer scale manufacturing. Such an electrically pumped low-noise ultra-dense frequency comb source is highly desirable in a number of applications, for example in high-resolution spectroscopy.

\section{Acknowledgements}

This work was carried out in the context of the European Research Council (ERC) starting grant ELECTRIC. S. C. and C. O. acknowledge the Flemish Research Council (FWO) for a Ph.D. scholarship. K. V. G. thanks the FWO for a postdoctoral fellowship.

\section{References}

[1] A. L. Gaeta, M. Lipson, and T. J. Kippenberg, "Photonicchip-based frequency combs", Nat. Photonics, vol. 13, no. 3, pp. 158-169, Mar. 2019.

[2] S. A. Diddams, K. Vahala, and T. Udem, "Optical frequency combs: Coherently uniting the electromagnetic spectrum", Science, vol. 369, no. 6501, 2020.

[3] K. Van Gasse, Z. Chen, E. Vicentini, J. Huh, S. Poelman, Z. Wang, G. Roelkens, T. W. Hansch, B. Kuyken, and N. Picque, "An on-chip III-Vsemiconductor-on-silicon laser frequency comb for gasphase molecular spectroscopy in real-time", Preprint at https://arxiv.org/abs/2006.15113, 2020.
[4] L. Consolino, M. Nafa, F. Cappelli, K. Garrasi, F. P. Mezzapesa, L. Li, A. G. Davies, E. H. Linfield, M. S. Vitiello, P. De Natale, and S. Bartalini, "Fully phase-stabilized quantum cascade laser frequency comb", Nat. Commun., vol. 10, no. 1, p. 2938, Jul. 2019.

[5] M. Zhang, B. Buscaino, C. Wang, A. Shams-Ansari, C. Reimer, R. Zhu, J. M. Kahn, and M. Lončar, "Broadband electro-optic frequency comb generation in a lithium niobate microring resonator", Nature, vol. 568, no. 7752, pp. 373-377, Apr. 2019.

[6] B. Stern, X. Ji, Y. Okawachi, A. L. Gaeta, and M. Lipson, "Battery-operated integrated frequency comb generator", Nature, vol. 562, no. 7727, pp. 401-405, Oct. 2018.

[7] N. Picqué and T. W. Hänsch, "Frequency comb spectroscopy", Nat. Photonics, vol. 13, no. 3, pp. 146-157, Mar. 2019.

[8] Z. Wang, K. Van Gasse, V. Moskalenko, S. Latkowski, E. Bente, B. Kuyken, and G. Roelkens, "A III-V-on-Si ultra-dense comb laser", Light Sci. Appl., vol. 6, no. 5, e16260-e16260, May 2017.

[9] K. Van Gasse, S. Uvin, V. Moskalenko, S. Latkowski, G. Roelkens, E. Bente, and B. kuyken, "Recent advances in the photonic integration of mode-locked laser diodes", IEEE Photon. Technol., vol. 31, no. 23, pp. 1870-1873, 2019.

[10] B. Haq, S. Kumari, K. Van Gasse, J. Zhang, A. Gocalinska, E. Pelucchi, B. Corbett, and G. Roelkens, "Microtransfer-printed III-V-on-silicon C-band semiconductor optical amplifiers", Laser Photonics Rev., vol. 14, no. 7, p. $1900364,2020$.

[11] C. Op de Beeck, B. Haq, L. Elsinger, A. Gocalinska, E. Pelucchi, B. Corbett, G. Roelkens, and B. Kuyken, "Heterogeneous III-V on silicon nitride amplifiers and lasers via microtransfer printing", Optica, vol. 7, no. 5 , pp. 386-393, May 2020.

[12] J. Justice, C. Bower, M. Meitl, M. B. Mooney, M. A. Gubbins, and B. Corbett, "Wafer-scale integration of group III-V lasers on silicon using transfer printing of epitaxial layers", Nat. Photonics, vol. 6, no. 9, pp. 610-614, Sep. 2012. 


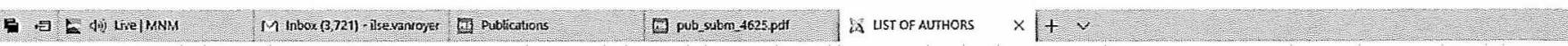

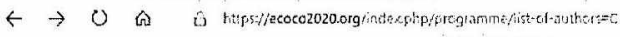

Borraccini, Giacomo (1): Straullu, Stefano (2): Ferrari, Alessio (1): Virgilito, Emànuele (1): Bottacchi, Stefano (3): Swall, scort (3): Piclaccia, Stefano (4): Galimberti, Gabriele (4) Gramnel. Gert (5): Curri. Vittorio (1)

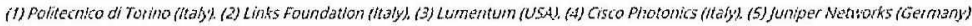

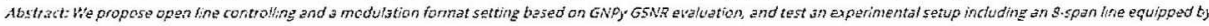

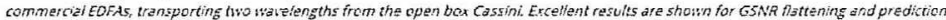

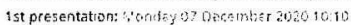

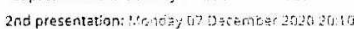

Curri. Vittorio

Thiti-4: Softwarized Optical Transport Qor in Production opticatNetwork: a Brownifield validation

Cumi. Vittorio (1); Ferrari, Alesslo (1); Bałasubramanian, Katthikeyan (2); Filer, Mark (2); Kundrat, Jan (3): Yin, Yäwei (2): Gramme1, Gert (4); Galimberv, Gabriele (5); Le Rouzic Esther (6)

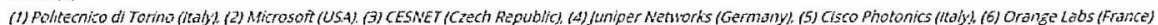

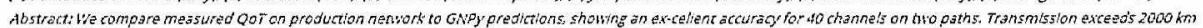

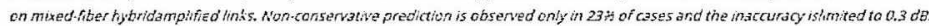

1st presentation: Thursjay 100 e cemibar $202003: 20$

2nd presentation: Fitratedy 10 ouseunber $202019: 30$

Cuyvers. 5tijn

Thit-A: (Highly scored) uttra-Dense III-V-on-Silicon Nitride Frequency Comb Laser

Cuyvers, Stijn (1): Haq. Bahawal (1): Op de Beeck, Camiel (2): Poelman, Stijn (2): Hermans, Artur (1): Wang, zheng (1): Roefkens. Gunther (2): Van Gasse, Kasper (1): Kuyken, Bart (2)

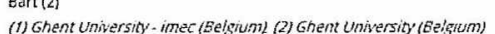

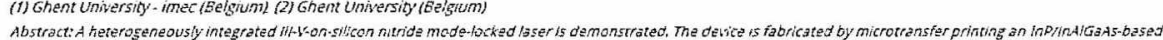

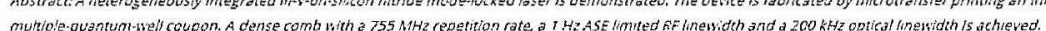

multiple quantul-swell coupon. A dense comb with a

2nd presentation: Thurces; 100 Desciniter $202013: 30$

da Cruz Jưnior.josè Hètio

Tu1f-4: Alt-Optical Nonlinear Pre-Compensation of Long-Reach Unrepeatered Systems

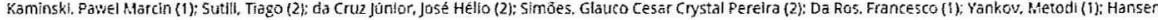
Hennik Enggazard (1): Clausent. Anders Thomas (1): Forctihammer, Ssren (1): Oxenlswe, Leif (1): Figueiredo, Rafael Carvalho (2): Gallh : sichaet (1)

(1) DTU Fotonit Tezthital University of Denamark (Oenmark) (2) Oplical Conmunications Division. CPQO (Brazil)

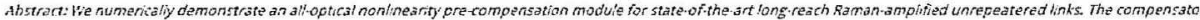

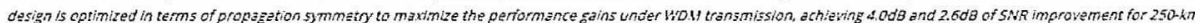
$3 n d 350$-km tinks.

Cookie policy

3 and 350 kn links.

2nd presentacon This 